\title{
Survival to admission after out-of-hospital cardiac arrest in Seoul, South Korea
}

\author{
This article was published in the following Dove Press journal: \\ Open Access Emergency Medicine \\ 2 September 2014 \\ Number of times this article has been viewed
}

\author{
Jin-Hue Kim' \\ Tai-Hwan Uhm² \\ 'Department of Emergency Medical \\ Technology, Sun Moon University, \\ Asan-si, Chungnam, South Korea; \\ ${ }^{2}$ Department of Emergency Medical \\ Services, Eulji University, Seongnam-si, \\ Gyeonggi-do, South Korea
}

Purpose: Out-of-hospital cardiac arrest (OHCA) data derived according to the Utstein Style guidelines was used to try to determine factors influencing survival to admission (STA) and epidemiological rates of OHCA.

Patients and methods: This was an observational study of all age groups based on data from prehospital care reports in Seoul, South Korea. The collected data were reported according to the Utstein Style template for OHCA and analyzed in order to compare STA with non-STA. Univariate analysis was conducted using a binomial logistic regression model to identify predictors associated with trauma patients.

Results: Eighty-three (4.8\%) OHCA survivors were admitted to the emergency department with carotid pulse. The median time from arrest to emergency medical personnel defibrillation was statistically significantly shorter in STA cases (8.0 minutes) than in non-STA cases (12.0 minutes; $P<0.001$ ). Factors independently associated with better prognosis in terms of trauma patients were female sex (odds ratio [OR]: $0.67 ; 95 \%$ confidence interval $[95 \% \mathrm{CI}]$ : 0.50-0.91; $P=0.01$ ), arrest at home (OR: 0.36; 95\% CI: $0.27-0.49 ; P<0.001)$, and witnessed arrest (OR: 2.64; 95\% CI: 1.94-3.39; $P<0.001$ ).

Conclusion: Early basic life support, performed by either a layperson or emergency medical personnel, had a positive effect on STA. Male sex, arrest outside of the home, and witnessed arrest are significantly associated with trauma.

Keywords: Utstein Style, prehospital, defibrillation, basic life support

\section{Introduction}

The outcomes of out-of-hospital cardiac arrests (OHCAs) are relatively poor in Korea. One reason for this is that most research to improve this problem has not precisely reflected the situation, due to differences between research based on different data sources and nonstandardized processes. To reduce such problems, the Utstein Style ${ }^{1}$ for OHCAs based on prehospital data has been adopted by a few researchers in Korea.

Survival rates of older trauma patients were lower as in other studies. ${ }^{1,2}$ However, survival to discharge rates of OHCA cases differ between different studies. Survival to admission (STA) rates in studies that were carried out based on in-hospital data were much higher than the rates in studies carried out based on out-of-hospital data. ${ }^{3-7}$ Therefore, it is difficult to compare outcomes between studies within Korea; ${ }^{3-7}$ however, witnessed arrest, witnessed cardiopulmonary resuscitation (CPR), witnessed automated external defibrillation (AED), and shockable rhythm were directly related to arrest place, witness and prehospital emergency treatments in Korean studies, and Korean studies ${ }^{3-7}$ revealed that some factors had a positive effect on outcomes in other studies. ${ }^{8-25}$
Correspondence: Tai-Hwan Uhm Department of Emergency Medical Services, Eulji University,

553 Sanseong-daero, Sujeong-gu, Seongnam-si, Gyeonggi-do,

South Korea

Tel +82 3I 7407258

Fax +82317407357

Email emtec@eulji.ac.kr 
The purpose of this study was to determine the factors influencing STA and OHCA rates according to the Utstein Style based on prehospital data. We hypothesized that early emergency treatment positively affects STA.

\section{Materials and methods}

This was an observational retrospective study based on data for all age groups in Seoul, South Korea from PCRs (prehospital care reports) and reports for cardiac arrest patients recorded by emergency medical technicians and reflecting the Utstein Style. For quality management, the 1,716 cases recorded on the PCRs and the reports for cardiac arrest patients according to the Utstein Style guidelines in Seoul from January 1 to May 31, 2013 were filed and input into a database. The data were collected according to the Utstein Style guidelines to improve quality of prehospital emergency care in Seoul Metropolitan Fire and Disaster Headquarters and the data were selected for this study by the Institute of Emergency Medical Services, Eulji University (Seongnam-si, South Korea).

In order to maintain confidentiality, the following were excluded from the report: 119 fire emergency centers; callers' names, phone numbers, and social security numbers; and guardians' names and addresses. The collected data were recorded and analyzed according to the Utstein Style template for reporting data on OHCA. Cardiac arrest was confirmed by emergency medical service (EMS) personnel in the case of carotid pulse absence, unresponsiveness, and apnea. Cardiogenic cardiac arrest was defined as the cessation of cardiac mechanical activity due to heart disease or presumed not to be of non-cardiac etiology, such as in cases of drug overdose, suicide, drowning, hypoxia, trauma, etc, by EMS personnel based on definitions in the Utstein Style. Bystander CPR was defined as chest compression provided by someone who is a layperson. STA was defined as a patient being transferred to the emergency department with carotid pulse and then admitted to hospital. Return of spontaneous circulation (ROSC) was prehospital return of any spontaneous palpable pulse.

The population of patients experiencing OHCA was characterized using the Utstein Style template. Continuous variables such as age and time intervals were compared via mean and standard deviation or median and interquartile range. Categorical variables including sex and site of arrest were expressed as percentages. In order to compare STA cases admitted to hospital with carotid pulse and non-STA, frequencies were compared using chi-square test, and continuous variables such as age and time intervals were compared by Student's $t$-test at a significance level of $P=0.05$. Univariate analysis was conducted using a binomial logistic regression model to identify predictors associated with trauma. Odds ratios (ORs) and 95\% confidence intervals (CIs) were calculated. The model calibration was evaluated by Hosmer-Lemeshow goodness-of-fit test. Statistical analysis was performed using SPSS for Windows (v 18.0; IBM Inc., New York, USA).

\section{Results}

The number of cardiac arrests and the rates according to the Utstein Style template are shown in Table 1. The Seoul EMS is likely to respond to about 4,118 OHCAs in a year, which was estimated from the collected 1,716 OHCAs between January 1 and May 31, 2013. The number of OHCAs for 12 months was calculated as 4,118 from 1,716 OHCAs over 5 months. Fifty-three cardiac arrests were donot-resuscitate cases, such as decomposition, rigor mortis, dependent lividity. A total of 1,663 patients (96.9\%) received resuscitation, including opening airway, giving oxygen, and chest compression by EMS personnel. Of the confirmed 1,663 cases, 341 cases (20.6\%) were of cardiac etiology, while the other cases had non-cardiac etiology including 295 trauma cases (17.2\%). 297 cases (17.3\%) were initial shockable rhythms (235 with ventricular fibrillation and 62 with ventricular tachycardia [VT]), while 1,419 cases were initial non-shockable rhythms (190 cases with asystole and 1,229 cases with the other rhythms according to the Utstein Style template). Three hundred and twenty three cases (18.8\%) were defibrillated (32 cases treated by laypersons and 291 cases treated by EMS personnel). There were 156 bystander CPR cases (9.1\%) and 83 STAs (4.8\%).

Table I OHCA rates according to the Utstein Style template

\begin{tabular}{lll}
\hline Template & N & Rate (\%) \\
\hline OHCA & 1,716 & \\
Resuscitation not attempted & 53 & \\
Cardiac etiology & 341 & 20.6 (cardiac etiology rate) \\
Trauma & 295 & 17.2 (trauma incidence rate) \\
Arrests witnessed (layperson) & 783 & 45.6 (witnessed arrests rate) \\
Arrests witnessed (EMS) & 140 & 8.2 (witnessed arrests rate) \\
Arrests not witnessed & 793 & \\
Initial VF & 235 & 17.3 (VF + VT rate) \\
Initial VT & 62 & \\
Initial asystole & 190 & \\
Other initial rhythms & 1,229 & \\
Bystander CPR & 156 & 9.1 (bystander CPR rate) \\
Defibrillation (layperson) & 32 & 1.9 (defibrillation rate) \\
Defibrillation (EMS) & 291 & 17.0 (defibrillation rate) \\
STA & 83 & 4.8 (STA rate) \\
\hline
\end{tabular}

Abbreviations: CPR, cardiopulmonary resuscitation; EMS, emergency medical services; OHCA, out-of-hospital cardiac arrest; STA, survival to admission; VF, ventricular fibrillation; VT, ventricular tachycardia. 
Table 2 Characteristics of out-of-hospital cardiac arrests transported to hospital by EMS

\begin{tabular}{|c|c|}
\hline Characteristics & $\mathbf{N}(\%)$ \\
\hline Mean age (years $\pm S D$ ) & $57.6 \pm 21.1$ \\
\hline \multicolumn{2}{|l|}{ Sex } \\
\hline Male & $\mathrm{I}, 06 \mathrm{I}(6 \mathrm{I} .8)$ \\
\hline Female & $655(38.2)$ \\
\hline \multicolumn{2}{|l|}{ Site of arrest } \\
\hline Home & $\mathrm{I}, 085(63.2)$ \\
\hline Residential area & $96(5.6)$ \\
\hline Road & $92(5.4)$ \\
\hline Local hospital & $40(2.3)$ \\
\hline River, sea, mountain & $27(1.6)$ \\
\hline Hotel & $22(1.3)$ \\
\hline Office & $22(1.3)$ \\
\hline Construction site & $15(0.9)$ \\
\hline School & $10(0.6)$ \\
\hline Other & $307(17.9)$ \\
\hline Minutes from arrest to bystander CPR (median \pm IQR) & $3.0 \pm 2.0$ \\
\hline Minutes from arrest to EMS personnel CPR (median \pm IQR) & $8.0 \pm 5.0$ \\
\hline $\begin{array}{l}\text { Minutes from arrest to EMS personnel defibrillation } \\
\text { (median } \pm I Q R \text { ) }\end{array}$ & $11.0 \pm 7.0$ \\
\hline
\end{tabular}

Note: Data are presented as $N(\%)$ unless otherwise specified. Abbreviations: CPR, cardiopulmonary resuscitation; EMS, emergency medical services; IQR, interquartile range; SD, standard deviation.

The number of patients transported to hospital by EMS personnel is reported in Table 2. The mean age of the patients was 57.6 years. There were more arrests among males $(61.8 \%)$ than females in the prehospital setting, and 1,085 (63.2\%) arrests occurred at home. The median time intervals were 3.0 minutes from arrest to bystander CPR; 8.0 minutes from arrest to EMS personnel CPR; and 11.0 minutes from arrest to EMS personnel defibrillation.

Univariate analysis of STA is presented in Table 3. The proportion of STA among the group of arrest at home was lower $(3.9 \% ; P=0.01)$ compared with that of outside of home (7.1\%). The proportion of STA among the group of witnessed arrest was higher $(8.6 \% ; P<0.001)$ compared with that of unwitnessed arrest. The proportion of STA among the group of layperson CPR was higher $(8.2 \% ; P<0.001)$ compared with that of non-layperson CPR. The proportion of STA among the group of layperson AED was higher $(25.0 \% ; P<0.001)$ compared with that of non-layperson AED. The proportion of STA among the group of EMS personnel defibrillation was higher $(13.2 \% ; P<0.001)$ compared with that of non-EMS personnel defibrillation. The proportion of STA among the group of trauma was lower $(1.7 \% ; P<0.01)$ compared with that of non-trauma $(5.8 \%)$. The median time from arrest to EMS personnel CPR was statistically significant. It was shorter in STA cases (7.0 minutes) than in non-STA cases
Table 3 Univariate analysis of STA predicted by variables

\begin{tabular}{|c|c|c|}
\hline Predictive variable & STA & $P$-value \\
\hline \multirow[t]{2}{*}{ Mean age (years $\pm S D$ ) } & Yes: $54.6 \pm 21.0$ & 0.23 \\
\hline & No: $57.8 \pm 21.0$ & \\
\hline \multicolumn{3}{|l|}{ Sex } \\
\hline Male & $53 / 1,008(5.3)$ & 0.70 \\
\hline Female & $30 / 625(4.8)$ & \\
\hline \multicolumn{3}{|l|}{ Arrest at home } \\
\hline Yes & $4 \mathrm{I} / \mathrm{I}, 044(3.9)$ & 0.01 \\
\hline No & $42 / 589(7.1)$ & \\
\hline \multicolumn{3}{|l|}{ Witnessed arrest } \\
\hline Yes & $67 / 716(8.6)$ & $<0.001$ \\
\hline No & $13 / 762(1.7)$ & \\
\hline \multicolumn{3}{|l|}{ Layperson CPR } \\
\hline Yes & $65 / 713(8.2)$ & $<0.001$ \\
\hline No & $17 / 865(1.9)$ & \\
\hline \multicolumn{3}{|l|}{ Layperson AED } \\
\hline Yes & $8 / 24(25.0)$ & $<0.001$ \\
\hline No & $74 / 1,593(4.4)$ & \\
\hline \multicolumn{3}{|l|}{ EMS defibrillation } \\
\hline Yes & $59 / 388(13.2)$ & $<0.001$ \\
\hline No & $24 / 1,245(1.9)$ & \\
\hline \multicolumn{3}{|l|}{ Trauma } \\
\hline Yes & $5 / 290(1.7)$ & 0.01 \\
\hline No & $78 / 1,343(5.8)$ & \\
\hline Minutes from arrest to bystander CPR & Yes: $2.0 \pm 2.0$ & 0.06 \\
\hline (median $\pm \mathrm{IQR})$ & No: $4.0 \pm 2.0$ & \\
\hline Minutes from arrest to EMS personnel & Yes: $7.0 \pm 5.0$ & $<0.001$ \\
\hline CPR (median \pm IQR) & No: $9.0 \pm 5.0$ & \\
\hline Minutes from arrest to EMS personnel & Yes: $8.0 \pm 6.0$ & $<0.001$ \\
\hline defibrillation (median $\pm I Q R$ ) & No: $12.0 \pm 8.0$ & \\
\hline
\end{tabular}

Note: Data are presented as $\mathrm{n} / \mathrm{N}$ (\%) unless otherwise specified.

Abbreviations: AED, automated external defibrillation; CPR, cardiopulmonary resuscitation; EMS, emergency medical services; IQR, interquartile range; SD, standard deviation; STA, survival to admission; $\mathrm{n} / \mathrm{N}$, number/total number.

(9.0 minutes; $P<0.001)$. The median time from arrest to EMS personnel defibrillation was statistically significant. It was shorter in STA cases (8.0 minutes) than in non-STA cases (12.0 minutes; $P<0.001$ ).

Logistic regression of trauma predicted by variables in 295 trauma cases is summarized in Table 4. There were significant independent predictors, such as female

Table 4 Logistic regression of trauma predicted by variables

\begin{tabular}{llll}
\hline Predictive variable & Odds ratio & $\begin{array}{l}\text { 95\% confidence } \\
\text { interval }\end{array}$ & P-value \\
\hline Age & 1.00 & $0.99-1.01$ & 0.79 \\
Sex (female) & 0.67 & $0.50-0.91$ & 0.01 \\
Site of arrest (home) & 0.36 & $0.27-0.49$ & $<0.001$ \\
Witness & 2.64 & $1.94-3.39$ & $<0.001$ \\
Bystander CPR & 1.12 & $0.82-1.51$ & 0.48 \\
Defibrillation & 1.57 & $1.09-2.27$ & 0.02 \\
Non-STA & 1.88 & $0.72-4.92$ & 0.20 \\
\hline
\end{tabular}

Note: Hosmer-Lemeshow goodness-of-fit test with chi-square $=5.20, P=0.740$. Abbreviations: CPR, cardiopulmonary resuscitation; STA, survival to admission. 
sex (OR: 0.67; 95\% CI: $0.50-0.91 ; P=0.01$ ), arrest at home (OR: $0.36 ; 95 \%$ CI: $0.27-0.49 ; P<0.001$ ), witnessed arrest (OR: 2.64; 95\% CI: 1.94-3.39; $P<0.001$ ), and defibrillation by EMS personnel (OR: 1.57; 95\% CI: 1.09-2.27; $P=0.02)$. Hosmer-Lemeshow goodness-of-fit test with chisquare $=5.20, P=0.740$ was used for the model calibration.

\section{Discussion}

Survival rates to discharge $(0.7 \%$ in case of trauma; $13.3 \%$ in case of infant; $6.5 \%$ in case of pediatric; $6.3 \%$ and $6.7 \%$ in case of adult; and $3.6 \%$ in case of geriatric) in Korea ${ }^{1,2,5-7}$ were similar to the trends in other countries' studies (Table 5). ${ }^{8,10,11,15-25}$ However, the $4.8 \%$ rate of STAs in this study was far lower than the STA rates of $23.3 \%-46.0 \%$ based on data drawn from the hospital care reports. We assumed the $4.8 \%$ STA rate via the prehospital data collected to be a more accurate result for OHCA, hospital records have data of only patients who came to the emergency centers, whereas our data were systematically collected from the Seoul Metropolitan Fire and Disaster Headquarters taking charge of prehospital EMS in Seoul.

The $4.8 \%$ STA rate is compared with the $7.6 \%$ rate found in a previous study in a similar urban area with a similar patient group, and patient definition. ${ }^{4}$ Nevertheless, the previous study based on PCR and hospital medical record differs from this study based on only PCR, and the study based on PCRs was well reflected OHCA compared with the other studies on HMR (see Table 5). Therefore, when all the Korean studies were combined, the prehospital STA rate of

Table 5 Survival to admission and survival to discharge in out-ofhospital cardiac arrest studies in Korea

\begin{tabular}{|c|c|c|c|c|c|}
\hline Area & Group & $\mathbf{N}$ & $\begin{array}{l}\text { Survival to } \\
\text { admission } \\
(\%)\end{array}$ & $\begin{array}{l}\text { Survival to } \\
\text { discharge } \\
\text { (\%) }\end{array}$ & $\begin{array}{l}\text { Data } \\
\text { source }\end{array}$ \\
\hline $\begin{array}{l}\text { Urban + } \\
\text { suburbs* }\end{array}$ & Whole & $|, 34|$ & 7.6 & NA & PCR \\
\hline Urban $^{4}$ & Whole & 5,526 & 7.6 & 4.5 & $\mathrm{PCR}+\mathrm{HMR}$ \\
\hline Urban $^{5}$ & Adult & 176 & 23.3 & 6.3 & HMR \\
\hline Urban ${ }^{6}$ & Adult & 449 & 41.2 & 6.7 & HMR \\
\hline Urban $^{2}$ & Geriatric & 804 & 46.0 & 3.6 & HMR \\
\hline Urban ${ }^{3}$ & Pediatric & 62 & 32.3 & 6.5 & HMR \\
\hline Urban $^{7}$ & Infant & 45 & 33.3 & 13.3 & HMR \\
\hline Urban' & Trauma & 409 & 35.2 & 0.7 & HMR \\
\hline
\end{tabular}

Notes: *Outcomes of out-of-hospital cardiac arrest in Gyeonggi, Korea. 'All pulseless patients with trauma for whom cardiopulmonary resuscitation was initiated. ${ }^{2}$ Geriatric who came to the emergency centers with non-traumatic out-ofhospital cardiac arrest. ${ }^{3}$ Pediatric who came to two tertiary hospitals were evaluated. ${ }^{4} \mathrm{H}$ ospital based cardiocerebrovascular disease monitoring. ${ }^{5} \mathrm{Clinical}$ analysis of prehospital cardiac arrest patients. ${ }^{6} \mathrm{Clinical}$ analysis of out-of-hospital cardiac arrest patients. ${ }^{7}$ Infant who arrived arrest at the emergency center of three hospitals. Abbreviations: HMR, hospital medical record; PCR, prehospital care report; NA, not available. all patient groups in urban areas could be considered to be approximately $5 \%$ in Korea.

Because cardiac etiology data were assessed by EMS personnel assessment based on non-postmortem cases, $20.6 \%$ of the data was probably underestimated. The $17.3 \%$ initial shockable rhythm was lower than the 19\% in Australia; $26 \%$ in Italy; $32 \%$ in New York city, USA; and 38\% in Amsterdam, the Netherlands, and this seemed to be caused by delayed emergency care. ${ }^{8,19,22,24}$ The American Heart Association have stated the chain of survival concept including early defibrillation for improving STA from sudden cardiac arrest. Early defibrillation remains crucial in VT and pulseless VT. To increase the chances of defibrillation delivery in sports facilities, train stations, and airports, and on trains, airplanes, passenger ships, etc, strategies of public-access defibrillation have been implemented since 2011 in Korea in attempts to reduce the time between patient arrest and defibrillation. However, the number of layperson defibrillations in this study (32 [1.9\%]) is still too low. Thus, more sophisticated strategies in terms of AED device placement and public education are required.

Arrest at home, witnessed arrest, layperson CPR and defibrillation, EMS personnel defibrillation, and short time to CPR and defibrillation all positively affected STA. Though layperson CPR and AED were verified to be contributing factors to STA, there was no statistically significant difference in the time to CPR between STA and non-STA; these results suggest that early CPR might not be a positive effect on STA, but high-quality CPR could have a positive effect on STA. It needs to be stressed to educate laypeople regarding effective chest compression techniques ("push hard and fast") given the current situation in Korea.

Median interval times, such as from arrest to EMS personnel CPR (7-9 minutes) and defibrillation (8-12 minutes), were also statistically significant. It was similar to survival discharge when definitive care within 8 minutes was delivered, whereas it was different to when CPR within 4 minutes was started in King County, WA, USA. ${ }^{15}$ In Asian studies, the call-to-shock times ranged from 9 to 11.7 minutes; this exceeded the arrest-to-shock time of 8 minutes. ${ }^{16-18}$ In the present study, collapse-to-shock times of 8 minutes in STA cases and 12 minutes in nonSTA cases were recorded by EMS personnel. In a metaanalysis, survival rates were constant when defibrillation time was less than 6 minutes. ${ }^{26}$ To improve the bystander CPR and AED rates should invigorate pre-arrival instructions by emergency medical dispatcher. This is supported by the findings of a previous study, in which the highest 
probability of survival was achieved when CPR was started by a bystander. ${ }^{22}$

We demonstrated that female sex (OR: 0.67), arrest at home (OR: 0.36), witnessed arrest (OR: 2.64), and EMS personnel defibrillation (OR: 1.57) were significant predictors of trauma in this study. These findings suggest that more males suffered serious wounds in accidents in public places such as roads, construction sites, and the work place. As a result, there were more witnesses of patient arrests.

However, bystander CPR to trauma had no statistically significant predictor, whereas EMS defibrillation had a statistically significant predictor. We speculated that laypersons hesitated to perform CPR due to dangers in the accident scene and bleeding of the patient; however, witnesses could recognize OHCA and called for EMS more quickly, and this had EMS personnel deliver more defibrillation for shockable rhythm.

\section{Conclusion}

Our study shows that the $4.8 \%$ STA rate derived from the prehospital data according to the Utstein Style guidelines is far lower than STA rates that were derived from hospital medical records selectively collected in OHCA cases. The overall rate of STA in this study is much lower than those in other developed nations. This is due to low rates of bystander CPR and defibrillation. Arrest site, witnessed arrest, layperson CPR, layperson defibrillation, EMS personnel defibrillation, and time to CPR, are significantly associated with STA. Male sex, arrest outside of the home, and witnessed arrest are significantly associated with trauma. Rates of survival to discharge and to 1 year were not assessed in this study, although these in-hospital outcomes will be necessary to inform EMS systems on effective strategies for improving survival rates following cardiac arrest.

\section{Acknowledgment}

This work was supported by the Sun Moon University Research Grant of 2012.

\section{Author contributions}

Both authors of this study made substantial contributions to the conception and design of the study, including analysis and interpretation of data; drafting the article and revising it critically for important intellectual content; and final approval of the version to be submitted. All contributors who do not meet the criteria for authorship as defined above are not listed.

\section{Disclosure}

The authors report no conflicts of interest in this work.

\section{References}

1. Kim YK, Lee KH, Lee SY, et al. [Resuscitation outcomes and clinical characteristics based on the initial ECG rhythm in prehospital traumatic cardiac arrest patients.] Journal of the Korean Society of Emergency Medicine. 2005;16:467-473. Korean.

2. Kim H, Kim SH, Oh SB, et al. [Resuscitation outcomes and clinical characteristics of non-traumatic out-of-hospital geriatric cardiac arrest.] Journal of the Korean Society of Emergency Medicine. 2004;15:434 439. Korean.

3. Yun SH, Lee KM, Kim JH, et al. [Outcome of pediatric out-of-hospital cardiac arrest] Journal of the Korean Society of Emergency Medicine. 2007;18:202-210. Korean.

4. Working Group of Emergency Physicians. 2007 Report on Hospital Based Cardiocerebrovascular Disease Registering and Monitoring System Construction. Seoul: Ministry of Health and Welfare; 2008. Korean.

5. Kim SJ, Cho SJ, Lee SL, Ryu SY, Kim HY, Kim SJ. [Impact of the level on the resuscitation prehospital cardiac arrest patients.] Journal of the Korean Society of Emergency Medicine. 2005;16:99-103. Korean.

6. Kim SE, Eo EK, Cheon YJ, Jung KY, Park HS. [Outcome in a tertiary emergency department for cardiopulmonary resuscitation for out-ofhospital cardiac arrest.] Journal of the Korean Society of Emergency Medicine. 2005;16:495-504. Korean.

7. Yun YY, Kim HJ, Han SC, et al. [Clinical analysis of CPR in infants with out-of-hospital cardiopulmonary arrest.] Journal of the Korean Society of Emergency Medicine. 2004;15:567-574. Korean.

8. Finn JC, Jacobs IG, Holman C.D'AJ, Oxer HF. Outcomes of out-ofhospital cardiac arrest patients in Perth, Western Australia, 1996-1999. Resuscitation. 2001;51:247-255.

9. Kämäräinen A, Virkkunen I, Yli-Hankala A, Silfvast T. Presumed futility in paramedic-treated out-of-hospital cardiac arrest: an Utstein style analysis in Tampere, Finland. Resuscitation. 2007;75:235-243.

10. Sirbaugh PE, Pepe PE, Shook JE, et al. A prospective, population-based study of the demographics, epidemiology, management, and outcome of out-of-hospital pediatric cardiopulmonary arrest. Annals of Emergency Medicine. 1999;33:174-184.

11. Stiell IG, Wells GA, DeMaio VJ, et al. Modifiable factors associated with improved cardiac arrest survival in a multicenter basic life support/defibrillation system: OPALS study phase I results. Annals of Emergency Medicine. 1999;33:44-50.

12. Olasveengen TM, Samdal M, Steen PA, Wik L, Sunde K. Progressing from initial non-shockable rhythms to a shockable rhythm is associated with improved outcome after out-of-hospital cardiac arrest. Resuscitation. 2009;80:24-29.

13. Cudnik MT, Schmicker RH, Vaillancourt C, et al; ROC Investigators. A geospatial assessment of transport distance and survival to discharge in out of hospital cardiac arrest patients: implications for resuscitation centers. Resuscitation. 2010;81:518-523.

14. Hess EP, Campbell RL, White RD. Epidemiology, trends, and outcome of out-of-hospital cardiac arrest of non-cardiac origin. Resuscitation. 2007;72:200-206.

15. Eisenberg MS, Cummins RO, Larsen MP. Numerators, denominators, and survival rates: reporting survival from out-of-hospital cardiac arrest. Resuscitation. 1991;9:544-546.

16. Leung LP, Lo CM, Tong HK. Prehospital resuscitation of out-ofhospital cardiac arrest in Queen Mary Hospital. Hong Kong Journal of Emergency Medicine. 2000;7:191-196.

17. Fan KL, Leung LP. Prognosis of patients with ventricular fibrillation in out-of-hospital cardiac arrest in Hong Kong: prospective study. Hong Kong Journal of Emergency Medicine. 2002;8:318-321.

18. Chan TH, Lui CT, Cheung KH, Tang YH, Tsui KL. Outcome predictors of patients in out-of-hospital cardiac arrests with pre-hospital defibrillation in Hong Kong. Hong Kong Journal of Emergency Medicine. 2013;20:131-137. 
19. Fairbanks RJ, Shah MN, Lerner EB, Ilangovan K, Pennington EC, Schneider SM. Epidemiology and outcomes of out-of-hospital cardiac arrest in Rochester, New York. Resuscitation. 2007;72:415-424.

20. Rewers M, Tilgreen RE, Crawford ME, Hjortsø NC. One-year survival after out-of-hospital cardiac in Copenhagen according to the 'Utstein style'. Resuscitation. 2000;47:137-146.

21. Dunne RB, Compton S, Zalenski RJ, Swor R, Welch R, Bock BF. Outcomes from out-of-hospital cardiac arrest in Detroit. Resuscitation. 2007;72:59-65.

22. Kette F, Sbrojavacca R, Rellini G, et al. Epidemiology and survival rate of out-of-hospital cardiac arrest in north-east Italy: The FACS study. Friuli Venezia Giulia Cardiac Arrest Cooperative Study. Resuscitation. 1998;36:153-159.

23. Engdahl J, Holmberg M, Karlson BW, Luepker R, Herlitz J. The epidemiology of out-of-hospital 'sudden' cardiac arrest. Resuscitation. 2002;52:235-245.
24. Waalewijn RA, de Vos R, Koster RW. Out-of-hospital cardiac arrests in Amsterdam and its surrounding areas: results from the Amsterdam resuscitation study (ARREST) in 'Utstein' style. Resuscitation. 1998;38:157-167.

25. Fredriksson M, Herlitz J, Nichol G. Variation in outcome in studies of out-of-hospital cardiac arrest: a review of studies conforming to the Utstein guidelines. Am J Emerg Med. 2005;21:276-281.

26. Nichol G, Stiell IG, Laupacis A, Pham B, De Mario VJ, Wells GA. A cumulative meta-analysis of the effectiveness of defibrillator-capable emergency medical services for victims of out-of-hospital cardiac arrest. Ann Emerg Med. 1999;34:517-525.

\section{Publish your work in this journal}

Open Access Emergency Medicine is an international, peer-reviewed, open access journal publishing original research, reports, editorials, reviews and commentaries on all aspects of emergency medicine. The manuscript management system is completely online and includes a very quick and fair peer-review system, which is all easy to use.
Visit http://www.dovepress.com/testimonials.php to read real quotes from published authors. 